\title{
BMJ Open Protocol for a meta-integration: investigating positive aspects of caregiving in dementia
}

\author{
Camille Branger, ${ }^{1}$ Megan E O'Connell, ${ }^{1}$ Shelley Peacock ${ }^{2}$
}

To cite: Branger $C$, O'Connell ME, Peacock S. Protocol for a metaintegration: investigating positive aspects of caregiving in dementia. BMJ Open 2018;8:e21215. doi:10.1136/ bmjopen-2017-021215

- Prepublication history and additional material for this paper are available online. To view these files, please visit the journal online (http://dx.doi org/10.1136/bmjopen-2017021215).

Received 18 December 2017 Revised 25 May 2018 Accepted 7 June 2018
Check for updates

(c) Author(s) (or their employer(s)) 2018. Re-use permitted under CC BY-NC. No commercial re-use. See rights and permissions. Published by BMJ.

${ }^{1}$ Department of Psychology, University of Saskatchewan, Saskatoon, Saskatchewan, Canada

${ }^{2}$ College of Nursing, University of Saskatchewan, Saskatoon, Saskatchewan, Canada

Correspondence to Dr Megan E 0'Connell; megan.oconnell@usask.ca

\begin{abstract}
Introduction The current work describes the protocol for a meta-integration investigating the positive aspects of providing care to someone living with dementia. We aim to understand the position of positive aspects in the caregiver experience as well as identify how positive aspects are commonly conceptualised, investigated and measured in literature.

Methods and analyses Meta-integration is a method of investigation that synthesises findings from metaanalysis or systematic review of quantitative studies and meta-synthesis of qualitative studies, to provide a coherent and holistic account of a phenomenon. As a relatively new method, terminology and methodological approaches are varied. The current work describes the process of conducting an advanced convergent metaintegration, including protocol for systematic search, inclusion/exclusion screening phases, intramethod analysis synthesis and intermethod synthesis on quantitative, qualitative and mixed methods research pertaining to the positive aspects of providing care to someone living with dementia.
\end{abstract}

Ethics and dissemination There are no ethics or safety concerns about dissemination, which includes plans for a conference presentation and publication.

\section{INTRODUCTION}

Advanced ageing is associated with an increased risk of developing dementia, a neurodegenerative disease that leads to increasing impairment in independent functioning and activities of daily living. ${ }^{1}$ Globally, it is estimated that 35.6 million people were living with dementia in 2010, and the prevalence of dementia is expected to rise as ageing populations continue to grow. ${ }^{2}$ Research indicates that care for persons living with dementia is largely provided by informal caregivers (ie, family members and friends; hereafter referred to as caregivers) who are untrained and unpaid for the care that they provide. ${ }^{2}$ Caregiving for a loved one with dementia poses challenges for caregivers financially, socially, ${ }^{3}$ physically and psychologically. ${ }^{4}$ Some research, however, has shown that there are positive aspects associated with caring for a loved one with dementia,

\section{Strengths and limitations of this study}

Meta-analysis of quantitative data may not be possible if methods used to measure positive aspects of caregiving vary across studies.

- Aggregative qualitative synthesis method might lose important contextual variable in the measurement of positive aspects of caregiving for persons with dementia, but this method is required to integrate quantitative and qualitative findings.

- Our broad approach to choosing search terms is a strength due to its comprehensiveness, but is labour intensive.

- Use of a single rater introduces possible biases in screening and data extraction, but our method includes use of a second coder in a small random sample to explore evidence for coder bias.

- This work will add to emerging field of meta-integration and the understudied positive aspects of caregiving.

including, but not limited to, feelings of personal gain and satisfaction. ${ }^{56}$ Research into the positive aspects of caregiving is growing, but it remains fraught with variations in labels and challenges in measurement and conceptualisation. Without a comprehensive understanding of what is currently known of positive aspects and how these aspects are conceptualised and measured, advances in salutogenic caregiver research are limited. Without a thorough understanding of the positive aspects of caregiving, supportive efforts of intervention programmes risk working to diminish negative aspects of caregiving while missing the opportunity to bolster positive aspects for caregivers. The researchers of the current work aim to gain a holistic account of the state of research on the positive aspects of caregiving through using a novel method of meta-integration which brings together findings from a meta-analysis of the quantitative studies pertaining to positive aspects of caring for a loved one with dementia and findings from a meta-synthesis of qualitative studies on the positive aspects of 
caregiving. The current work describes the protocol for the meta-integration.

\section{Research question}

The researchers propose that meta-integration would be central to advancing the study of positive aspects of caregiving for persons with dementia. Meta-integration is the chosen method for this objective because this method can determine the current status of positive aspects in quantitative and qualitative research, and by integrating varied approaches to studying positive aspects of caregiving we hope to gain a cohesive understanding of the phenomena. The research questions for current meta-integration are: (1) how are positive aspects of caregiving most commonly conceptualised in research? (2) what are the most common labels used to refer to positive aspects of caregiving? (3) what are the most common methods of measuring positive aspects of caregiving? (4) what do the data indicate about the relationship between positive aspects and other informal caregiving variables?

\section{METHOD}

Meta-integration, or mixed-meta-synthesis, is a novel form of research synthesis that integrates quantitative meta-analysis and qualitative meta-synthesis. Differences in ontological and epistemological perspectives historically divided quantitative and qualitative research. ${ }^{7}$ Each approach, however, has strengths. Meta-analysis is the classic method for aggregating related empirical findings and can generate new knowledge and has proved useful in the development of theory. ${ }^{89}$ Meta-analysis consists of two main parts: a systematic review of literature of a particular field of study/research question (eg, caregiver satisfaction and caregiver well-being) and high order statistical analysis of these literature findings. ${ }^{89}$ For qualitative data, meta-synthesis can reveal powerful explanations, provide greater generalisability and increased levels of abstraction ${ }^{10}$ which allows researchers to revise, or refute, extant theories and understanding of human phenomenon. ${ }^{11}$ Meta-synthesis methods can be aggregative or interpretive. Aggregative meta-synthesis methods use the findings from systematically reviewing the qualitative literature to identify themes or similar descriptors in order to produce a general description of the phenomenon under study. ${ }^{11}$ Aggregative synthesis methods (eg, meta-summary, thematic analysis, content analysis, case survey, qualitative comparative analysis and Bayesian meta-analysis) do not consider the context under which individual study findings occur. ${ }^{11}$ In contrast to interpretive meta-synthesis methods that involve a high degree of circular iteration for analysis, aggregative models are marked by low or absent iteration and, instead, adopt a highly structured manner of selecting, organising and reporting individual study findings. ${ }^{11}$

Meta-integration methods can be categorised as segregated or integrated. ${ }^{7}$ Segregated, or convergent, designs assume that quantitative and qualitative studies, and related findings are different entities that must be treated separately. Thus, a segregated method is suitable when the synthesis outcome is intended to be a configuration, not assimilation of the research findings. ${ }^{7}$ Integrated designs, conversely, do not view quantitative and qualitative research approaches as fundamentally different, but rather as producing findings that are easily transformed from quantitative to qualitative and vice versa. ${ }^{712}$ Thus, integrated designs are suitable when synthesis is intended to produce assimilated research findings. ${ }^{7}$ The current research endeavour is exploratory, consequently we concluded that a configuration of the findings (ie, segregated $^{8}$ or convergent ${ }^{13}$ ) would be more appropriate than assimilation. ${ }^{712}$

To design the current protocol, we relied on the models of meta-integration described by Frantzen and Fetters. ${ }^{13}$ Frantzen and Fetters ${ }^{13}$ compared published methods of synthesising work from quantitative, qualitative and mixed-method studies. Models of meta-integration are differentiated by the inclusion or exclusion of mixedmethods studies (inclusion of mixed-methods require advanced model designs, rather than basic models designs) and use of data transformation (where, for example, a researcher might choose to transform quantitative data into qualitative data and conduct a convergent qualitative meta-integration). We chose an advanced model over the basic model due to the inclusion of mixed-method studies. Further, given the exploratory nature of this research, we determined that models of integration that included data transforming (transforming quantitative data into qualitative data and vice versa for synthesis purposes) would be inappropriate, as we did not want to privilege any one line of inquiry (ie, quantitative or qualitative). In the chosen model of meta-integration, the mixed methods studies are fractionated, that is, quantitative data and qualitative data are extracted and added to quantitative and qualitative datasets, respectively. After this step, we integrate the findings using intermethod synthesis.

The following outlines the protocol for conducting the advanced convergent meta-integration, including the process of determining the research question(s), conducting the systematic search, inclusion and exclusion screening process, intramethod synthesis-analysis, intermethod synthesis and, finally, organisation of results, assessment of fit and conclusions.

\section{Advanced meta-integration protocol}

1. Categorise studies

2. Identify literature (see systematic literature search protocol below)

a. Divide studies into quantitative, qualitative and mixed method categories

b. Fractionate mixed methods studies

i. Extract quantitative data from mixed method studies and add to quantitative dataset, extract qualitative data from mixed methods studies and add to qualitative data set.

c. Coding and data extraction 
i. Develop coding manual and develop coding summary sheet.

ii. $10 \%-15 \%$ of final references coded by second coder to assess consistency and potential bias. If evidence for bias exists, a second coder will have to be used consistently and consensus in coding will be required.

iii. Extract data and document on code summary sheet.

iv. Create database of coded reference material.

3. Conduct intramethod analysis-synthesis and comparison

a. Intramethod analysis of quantitative dataset.

b. Intramethod analysis of qualitative dataset.

4. Conduct intermethod integration

5. Organise results and assess fit

6. Draw final conclusions.

\section{Step 1: Systematic literature search}

The following steps outline the approach used to obtain the sample of references to be used in the proposed meta-integration:

1. Identify relevant databases

2. Identify search terms

a. Develop concept charts for each database

3. Conduct search of each database

a. Export each database search results into reference manager programme

4. Screening criteria

a. Develop protocol to determine eligibility for inclusion in study

i. Based on protocol most appropriate for research endeavour (eg, PICO, SPICE, SPIDER, among others)

5. Phase I screening

a. Screen titles and abstract for relevance

6. Phase II screening

a. Screen methods and measures for relevance.

7. Final screening phase

a. Screen body of reference for relevance.

b. Organise retained references by scientific approach (ie, quantitative studies, qualitative studies, mixed-method studies).

c. Document inclusion and exclusion.

\section{Search strategy: Step 1}

A systematic literature search for relevant studies will be conducted using the databases: PsychINFO, MEDLINE, Cumulative Index of Nursing and Allied Health Literature (CINAHL), Sociological Abstract, SocINDEX, AgeLine, Anthropology plus and Embase. Databases were chosen in consultation with a librarian employed at the University of Saskatchewan who specialises in psychology and selection was based largely on relevance (ie, disciplines likely to have interest/investigation in the topic of caregiving and dementia). The method of selection is in line with Crumley and Blackhall's ${ }^{14}$ guide on search strategies for systematic reviews.

\section{Concept chart development: Step 2}

The researchers chose 'caregiver,' 'dementia' and 'positive aspects' to be the search concepts used for the systematic search (these concepts were chosen based on the researchers knowledge of the most common labels used to described these constructs, being active researchers in the area). Synonyms for each of these search terms will be identified for each database and included in the search. To assist this aim, a 'concept chart' will be created for each database. Disciplines differ in the terms they use to describe concepts, thus synonyms for the search term had to be identified in each database. For example, we will enter search term 'caregiver' in the database PsychINFO (Ovid platform). We will search the key word and subject heading sections of the first 100 or more references of 23012 returned (the decision to stop will be somewhat arbitrary and based on repetition of the identified synonyms and marked decrease in identification of new synonyms). Next, a search of PsychINFO 'caregiver' AND 'dementia' ('exploded' to include 'dementia' derivatives) will be entered to ensure that any additional synonyms for caregivers in the context of dementia may be captured. The first 100 or more of the 3296 articles returned will be searched and new synonyms added. A new search for synonyms for the construct 'dementia' will be completed and 100 or more articles of the 28466 returned will be searched for dementia synonyms.

The process will be repeated for the search term 'positive aspects'. Having experience in this research area, the researchers understand that many terms have been used to describe positive aspects, thus identification of all possible synonyms is particularly important in identifying relevant articles. Positive aspects in the context of caregiving will be searched (positive aspects (as keyword) AND caregiving ('exploded to include derivatives of the term)). All articles will be searched and synonyms for 'positive aspects' identified. Please see table 1 for concept chart exemplar. The researchers will conduct the search and examine the identified references to assess whether the articles returned are relevant to the current research endeavour. The researchers will be able to identify 'synonyms' that result in references that are not appropriate for the current research study. For instance, a positive aspects synonym, 'psychological endurance', may be found to pertain to negative aspects of caregiving, rather than positive. Similarly, another synonym, 'quality of care', may be found to be too vague and related to professional caregivers. The synonyms identified as not appropriate for the search will be indicated on the concept chart with notation regarding reason for omission (table 1).

The PsychINFO concept chart will be comprehensive and will be used as a general concept chart to be adapted to all other databases. More specifically, we will repeat the process of identifying synonyms for each concept in each database and those unique to the particular database will be added to the general concept chart (synonyms unique to the database will be indicated on concept chart by italicised font). 
Table 1 Search term synonyms identified in the Psychlnfo database

\section{General (Psychlnfo)}

Concept Chart

\begin{tabular}{|c|c|c|}
\hline $\begin{array}{l}\text { Concept A } \\
\text { Caregiver (16) }\end{array}$ & $\begin{array}{l}\text { Concept B } \\
\text { Dementia (21) }\end{array}$ & $\begin{array}{l}\text { Concept C } \\
\text { Positive Aspects (36) }\end{array}$ \\
\hline Care partner* & AIDS dementia complex/ & Adaptability- too vague \\
\hline Care provider * & Alzheimer's disease/ & Advocacy-too vague \\
\hline Caregivers/ & Cognitive decline & Assertiveness-to vague \\
\hline Carer $^{*}$ & Cognitive impairment/ & Autonomy/ \\
\hline Caring behavior* & Corticobasal degeneration/ & Behavioural intention-too vague \\
\hline Child caregiv* & Creutzfeldt Jakob syndrome/ & Caregiving benefit* \\
\hline Elder care/ & Dementia with Lewy bodies/ & Caregiving competence \\
\hline Familial care $^{*}$ & Dementia*/ & Caregiving gain* \\
\hline Family care partner*-redundant & Dysexecutive syndrome- redundant & Contentment/ \\
\hline Family care provider-redundant & Early onset dementia & Coping behaviour-too broad \\
\hline Family care* & Frontotemporal dementia & Emotional adjustment- not positive \\
\hline Informal caregiv* & Frontotemporal lobar degeneration & Empowerment \\
\hline Informal care* & FTD- acronym, redundant & Enthusiasm-Too broad \\
\hline Primary caregiv* & Late onset dementia & External reward/ \\
\hline Primary family caregiv* & Memory disorders/ & Finding meaning \\
\hline Quality of care/ - too vague & Neurodegeneration/ & Gain* \\
\hline $\begin{array}{l}\text { Sandwich generation caregiver*- } \\
\text { redundant }\end{array}$ & Neurodegenerative diseases/ & Independence--Too broad \\
\hline \multirow[t]{24}{*}{ Spouse caregiver* } & Parkinson's dementia & Intention-- Too broad \\
\hline & Picks disease/ & Internal reward/ \\
\hline & Presenile dementia- not relevant & Intrinsic behavior-not relevant \\
\hline & Semantic dementia/ & Intrinsic motivation \\
\hline & Senile dementia/ & Involvement- too vague \\
\hline & Vascular dementia & Life satisfaction/ \\
\hline & Young onset dementia & Meaning/ \\
\hline & & Meaningfulness/ \\
\hline & & Motivation/ \\
\hline & & Optimism/ \\
\hline & & Persistence/ \\
\hline & & Positive aspect ${ }^{\star}$ \\
\hline & & Positive caregiver experience* \\
\hline & & Positive caregiver outcome* \\
\hline & & Positive dimension* \\
\hline & & Positive emotion* \\
\hline & & Positive feeling* \\
\hline & & Positive psychology/ \\
\hline & & Positive value-too vague \\
\hline & & Positivism-not relevant \\
\hline & & Posttraumatic growth/ \\
\hline & & Protective factors-too vague \\
\hline & & Psychological Endurance - infers negative \\
\hline & & $\begin{array}{l}\text { Psychological Stress _not } \\
\text { Positive=omitted }\end{array}$ \\
\hline
\end{tabular}


Table 1 Continued

\section{General (PsychInfo)}

Concept Chart

\begin{tabular}{ll}
\hline Concept A & Concept B \\
Caregiver (16) & Dementia (21) \\
\hline
\end{tabular}

Concept C

Positive Aspects (36)

Quality of life-too vague/not relevant

Relationship satisfaction

Resilience/(psychological)

Rewards/

Role satisfaction/

Satisfaction/

Self-affirmation

Self-confidence/

Self-determination/

Self-efficacy/

Self-evaluation/

\section{Self-perception/}

Well-being/

Terms that were searched as subject heading are indicated with ' $\%$. The asterisk notation indicates truncation and the search would include that term with any suffix (eg, caregiv* includes, caregiver, caregiving). Items in bold formatting were items included in final search, unbolded items were omitted.

\section{Database search: Step 3}

Once concept charts for each database are completed, the systematic search will commence. No limit in terms of year of publication will be placed on the search. While the stated goal is to understand the current conceptualisation and measurement of positive aspects, the researchers understand that this is a relatively new area of research and identified studies are unlikely to be outdated to the point where they are not of use. In addition, gaining a sense of the history and evolution of the concept and its measurement should prove to elucidate the current state of the concept. In each database, the term 'caregiver' and all identified synonyms will entered, using OR action (eg, 'caregiv*' OR 'care partner' OR and so on). Then the term 'dementia' and all identified synonyms will be entered, using OR action. The term 'positive aspect*' and all identified synonyms will be entered using OR action. In addition, each term's 'exploded' derivatives will be considered for inclusion. The three compiled search terms (ie, including all synonyms) will be searched together using AND action. References from each database will exported into their respective folders in Zotero reference manager.

\section{Screening criteria: Step 4}

The PICO and SPIDER guides will be used. The search tools, PICO and SPIDER, have been shown to have good sensitivity and specificity, respectively, in identifying relevant references. ${ }^{15}$ As noted, the researchers used a liberal search approach, thus we rely on the guidance of PICO and SPIDER later in the screening phase (ie, eligibility phase), rather than reference identification phase. Given its applicability to quantitative research design, ${ }^{15}$ PICO will be used to inform criteria pertaining to quantitative studies. SPIDER protocol is suitable for reviews with an exploratory nature and will guide the majority of the eligibility criteria as it is applicable to quantitative, qualitative and mixed method studies ${ }^{16}$ (see online supplementary appendix A for eligibility protocol).

\section{Screening for inclusion: Steps 5 and 6}

Screening phase I

In the first screening phase, titles and abstracts will be screened to determine whether the manuscript pertains to positive aspects and informal caregivers of persons diagnosed with dementia or mild cognitive impairment (MCI; although it is meant to describe a condition with no functional impairment, the researchers understand that the term MCI is used inconsistently and may be applied to those who do require some assistance, thus, the researchers chose to err on the side of caution and 'cast a wide net' by including MCI). If unclear based on title, abstracts will be screened to ensure that the study relates to informal caregiver and experience/caregiver outcomes and/or positive aspects/synonyms. If the abstract does not include 'positive aspects' or a synonym, but does address caregiver non-negative outcomes/experience, the article will be kept. If it is unclear, the article will be kept for further screening.

\section{Screening phase II}

In the second screening phase, the methods and measures sections of manuscripts will be examined. For inclusion, the study must report positive aspects/synonyms 
or clearly report on non-negative caregiver experience (ie, not exclusive to caregiver: burden, burnout, distress, strain, negative health effects). The positive aspect has to be measured and described. The inclusion criterion was purposefully broad because labels and definition of positive aspects are currently inconsistent in the burgeoning field of positive aspects in caregiving for someone with dementia.

\section{Final screening phase}

After phase I and II screening, the full body texts of remaining references will be screened for eligibility using the eligibility criteria (online supplementary appendix A). References excluded in this phase will be documented, including reason for exclusion. The references will be divided into quantitative, qualitative and mixed-methods categories before data extraction and coding phase.

\section{Categorise studies: Step 7}

Development of the coding manual will first be based on theory and will be amended during data extraction phase. Due to the exploratory nature of the research endeavour, the researcher may find predetermined variables are not applicable (ie, not investigated/reported in primary studies) and new variables of interest might emerge (ie, primary studies might present novel constructs related to positive aspects or new facets of positive aspects). The Mixed Methods Appraisal Tool (MMAT) was designed for the appraisal stage of systematic literature reviews that include quantitative, qualitative and mixed-methods studies. ${ }^{17}$ The MMAT will be used to describe the methodological quality of each study, and the MMAT score for each study will be included in the database. The following variables from primary studies will be extracted (online supplementary appendix B).

\section{Study variables}

Study identification number, publication type (journal article, thesis/dissertation), publication year, care-recipient dwelling (eg, community, institution and so on) and study country of origin.

\section{Research variables}

Purpose of the study, relationships measured (ie, positive aspects in relation to caregiver age, burden and so on), caregiver sample size, care-recipient characteristics (ie, persons diagnosed with dementia, dementia due to Alzheimer's disease and so on), caregiver/care-recipient relationship, number of caregiver relationship types (ie, number of spousal caregivers in sample, number of child caregivers in sample and so on), mean age of caregivers, number of females in caregiver sample, number of males in caregiver sample, number of caregivers employed, sample mean duration of caregiving in years.

\section{Study design variables}

Theoretical approach, stated epistemology, study design.

\section{Construct variables}

Labels given to describe positive aspects of caregiving, positive aspects definition, positive aspects measure(s), properties of positive aspects measure(s), evidence of validity and reliability of positive aspects measure(s), sample size, mean and SD on positive aspects of caregiving measure. Relationship between positive aspects scores and caregiver: sex, age, race, relationship to care-recipient, employment, care-recipient dementia diagnosis, years spent caregiving, burden, distress, psychological health/well-being, physical health/well-being, subjective health, satisfaction with life, coping style, support, level of education, religiosity/spirituality, competence/mastery/ self-efficacy, quality of caregiver/care-recipient relationship and severity of care-recipient dementia, dementia behaviours and symptoms.

After data extraction of a primary study is complete, the reference section will be cross-referenced against the sample of studies for the meta-integration and new relevant references will be obtained and added to either the quantitative, qualitative or mixed-methods sample pools.

\section{Conduct intramethod analysis-synthesis and comparison: Step 8}

The intramethod analysis and synthesis is an iterative process where the quantitative dataset is synthesised and analysed and the qualitative dataset is synthesised and analysed, ensuring a separate overview is created for each dataset. ${ }^{13}$ Synthesis will bring together the main findings from each dataset, combining the results and interpretations to create an integrated and summative account of all the quantitative studies and then of all the qualitative studies. The iterative synthesis and analytic process will occur simultaneously, as results and interpretations of the data will be deconstructed and reconstructed, separated into their previous state and then recombined. The iterative synthesis-analysis process is what will allow for new perceptions and advancements in knowledge of the phenomenon to occur. ${ }^{13}$ The current protocol is elaborated in the following sections, quantitative dataset synthesis and qualitative dataset synthesis.

\section{Quantitative dataset synthesis}

The extracted data from quantitative studies will be closely reviewed to gain a sense of the degree of homogeneity. Specifically, we will look at whether there is enough consistency in the measures used for positive aspects across studies (ie, it would not be appropriate to group measures of gain with measures of satisfaction) and in the variables in which positive aspects are investigated in relation to (ie, it would not be appropriate to group investigation of positive aspects and caregiver burden with positive aspect and caregiver distress), to calculate effect sizes. It is possible that the studies will be too few and perhaps too heterogeneous in methods of measurement and variables investigated for meta-analysis. If the studies do not vary greatly on the factors described above, the variables of interest will be extracted, coded 
and findings from the studies will be transformed into a common metric to calculate an overall effect size for the relationship(s) of interest. ${ }^{18}$ Should a meta-analysis not be possible, a narrative analysis of the primary studies will be conducted. A narrative analysis will summarise and bring together the findings from primary studies and may employ frequency counts or other quasi-statistical approaches to best describe the meta-data. ${ }^{8}$ Both meta-analysis and narrative analysis are appropriate for meta-integration methods. ${ }^{7}$

\section{Qualitative dataset synthesis}

The qualitative studies will be closely reviewed and the following data will be extracted and included in the dataset: author(s), epistemology, sample size, sample population, aims/topics, method of data collection, data analysis, findings and notes/caveats. The researchers will use thematic synthesis of the qualitative dataset, as described by Kavanagh and colleagues. ${ }^{19}$ Qualitative data to be included in the dataset can be part of the body of text, and Kavanagh et $a l^{19}$ suggest the 'findings' or 'results' sections of primary studies be used as the raw data to be extracted. Should a study's goals correspond to the current research study, then the conclusions drawn by the primary study may be included in the dataset. ${ }^{19}$ Primary study findings will be summarised in data extraction form and included in the dataset and consideration will be given to the ways in which the methodologies and epistemologies used in the primary study shaped the understandings and findings. Line by line coding of the dataset will be conducted. Each line will receive a code that encapsulates the meaning. Conceptual translation is a key characteristic of qualitative synthesis and it occurs when codes begin to be applied to data from a second primary source. ${ }^{19}$ Either performed simultaneously or after line by line coding, the researcher(s) will generate descriptive codes and organise the emerging codes into descriptive themes. To do so, the researcher will develop an overarching conceptual framework to group codes that are conceptually similar. While the development of the framework will require some interpretation, the purpose is to summarise and organise the dataset rather than draw new/original conclusions.

Importantly, while the two datasets (quantitative and qualitative) are not yet integrated at the intramethod synthesis-analysis stage, it is unrealistic to suggest that the researcher can effectively disentangle pondering the emerging findings from the two separate datasets. The synthesis-analysis of quantitative dataset will be affected by the synthesis-analysis of the qualitative dataset, and vice versa, even if only at the subconscious level of the researcher. Thus, the model includes the process of 'mindful comparison' during the synthesis-analysis of each dataset. ${ }^{13}$ Mindful comparison describes the conscious and intentional consideration of the findings of each dataset, paying heed to the similarities and differences between the quantitative and qualitative datasets and, ultimately, how they relate to one another. The mindful comparison process lays the intellectual groundwork for the intermethod synthesis. ${ }^{13}$

\section{Conduct Intermethod integration: Step 9}

The intermethod integration will be a gradual iterative transition from intramethod synthesis-analysis to intermethod integration. Although an iterative transition, it will be important to have 'completed' the intramethod synthesis-analysis of each database before transitioning into the intermethod integration, as a thorough understanding of each data set (for instance, consistent themes, relationships investigated, main findings) is important before integrating the two. ${ }^{13}$ To facilitate intermethod integration, displays of each dataset will be created; these 'joint displays' will provide an overview of the complex inter-relational connections within each dataset and facilitate identification of connections across datasets. The 'joint displays' may take many forms and will likely go through much iteration, and refinement, as insights about the data emerge. The 'joint displays' are thought to support and foster a better understanding of the dissimilar data during the analytic phase, but are also useful in the dissemination of results in final publications and presentations.

\section{Organise and assess fit: Step 10}

In this phase, organisation refers to the final and comprehensive grouping of the data for presentation of the end product. ${ }^{13}$ Importantly, the organisation process includes backtracking and determining the origins of the data; this is a critical part of the process that allows for full clarity and accountability. In backtracking, the researcher will make a constant effort to keep track of the underpinnings, or statements, that lead to each conclusion.

\section{The final stage of organisation will be an assessment of 'fit'}

'Fit' refers to examining the concordance between the finding of the integrated datasets. ${ }^{20}$ Here, similarities in results and conclusions across the two databases indicates support. If the findings contradict each other, there is discordance. If the findings address different aspects of the phenomenon, there is expansion. The researcher will reflect on the 'fit' and make arguments for the 'fit' of the integration, which is expected to strengthen the quality of the conclusions. Outcomes from 'fit' assessment may also serve to inform future research directions.

\section{Draw final conclusions: Step 11}

After completing the processes of synthesis-analysis, integration, organisation and 'fit', the researcher will draw final conclusions based on all the included sample studies. The conclusions will go beyond reiteration of specific findings to focus on novel information and knowledge based on the findings from the convergent meta-integration findings. 


\section{Patient and public involvement}

This is a review of published material; consequently, for the current project there was no patient or public involvement.

\section{Ethics and dissemination}

There are no ethical or safety concerns associated with the proposed research. No participants will be used in this study. The findings from this research will be published in scholarly journals and will be available through the Alzheimer's society of Canada, the funding source of the graduate student's research.

\section{CONCLUSION}

This paper describes protocol for conducting a meta-integration, which is a relatively novel method of investigation of quantitative and qualitative inquiry to provide a coherent and holistic account of a particular phenomenon. ${ }^{19}$ Literature on caregivers of persons living with dementia focuses predominantly on negative aspects of caregiving, but increasingly, the positive aspects of caregiving are being studied. As a new area of inquiry, however, the study of positive aspects of caring for persons with dementia displays variation in labels and definitions of positive aspects. The current work describes the protocol used to conduct a meta-integration on literature pertaining to positive aspect so caregiving with the aim of identifying common labels and conceptualisations, common measures and relationships between positive aspects and other caregiving-related factors.

Contributors $\mathrm{CB}$ is the primary author of this work, but MEO'C revised the manuscript post-peer review. MEO'C and SP served to review and revised the document. The guarantor of this review is MEO'C.

Funding This work was supported by a doctoral award provided by the Alzheimer Society of Canada to CB. The Alzheimer Society of Canada was not involved in the development of this protocol.

Competing interests None declared.

Patient consent Not required.

Provenance and peer review Not commissioned; externally peer reviewed.

Data sharing statement We can provide summary data.

Open access This is an open access article distributed in accordance with the Creative Commons Attribution Non Commercial (CC BY-NC 4.0) license, which permits others to distribute, remix, adapt, build upon this work non-commercially, and license their derivative works on different terms, provided the original work is properly cited, appropriate credit is given, any changes made indicated, and the use is non-commercial. See: http://creativecommons.org/licenses/by-nc/4.0/.

\section{REFERENCES}

1. Lezak MD, Howieson DB, Bigler ED, et al. Neuropsychological assessment. 5th Edn. Oxford: Oxford University Press, 2012.

2. Prince M, Bryce R, Albanese E, et al. The global prevalence of dementia: a systematic review and metaanalysis. Alzheimers Dement 2013;9:63-75.

3. Brodaty $\mathrm{H}$. Meaning and measurement of caregiver outcomes. Int Psychogeriatr 2007;19:363-81.

4. Pinquart M, Sörensen S. Differences between caregivers and noncaregivers in psychological health and physical health: a metaanalysis. Psychol Aging 2003;18:250-67.

5. Farran CJ. Theoretical perspectives concerning positive aspects of caring for elderly persons with dementia: stress/adaptation and existentialism. Gerontologist 1997;37:250-7.

6. Peacock S, Forbes D, Markle-Reid M, et al. The positive aspects of the caregiving journey with dementia: using a strengths-based perspective to reveal opportunities. Journal of Applied Gerontology 2010;29:640-59.

7. Sandelowski M, Voils $\mathrm{Cl}$, Barroso J. Defining and designing mixed research synthesis Studies. Res Sch 2006;13:29.

8. Cooper H. Research synthesis and meta-analysis: a step-by-step approach. 4th Edn. Los Angeles: Sage Publications, 2009.

9. Schulze R. Meta-analysis: a comparison of approaches. Cambridge, MA: Hogrefe \& Huber Publishers, 2004.

10. Sherwood G. Meta-synthesis: Merging qualitative studies to develop nursing knowledge. International Journal for Human Caring 1999;3:37-42.

11. Hannes K, Lockwood C. Synthesizing qualitative research: choosing the right approach. Chichester, UK: John Wiley \& Sons, 2011.

12. Onwuegbuzie AJ, Teddlie C. A framework for analyzing data in mixed methods research. In: Tashakkori A, Teddlie C, eds. Handbook of mixed methods in social and behavioral research. Thousand Oaks: Sage Publications, 2003:397-430.

13. Frantzen KK, Fetters MD. Meta-integration for synthesizing data in a systematic mixed studies review: insights from research on autism spectrum disorder. Qual Quant 2016;50:2251-77.

14. Crumley E, Blackhall K. Setting up search strategies for systematic reviews (or, how many ways can you spell diarrhea?). Bibliotheca Medica Canadiana 2003;24:167-8.

15. Methley AM, Campbell S, Chew-Graham C, et al. PICO, PICOS and SPIDER: a comparison study of specificity and sensitivity in three search tools for qualitative systematic reviews. BMC Health Serv Res 2014;14:579.

16. Cooke A, Smith D, Booth A. Beyond PICO: the SPIDER tool for qualitative evidence synthesis. Qual Health Res 2012;22:1435-43.

17. Pace R, Pluye P, Bartlett G, et al. Testing the reliability and efficiency of the pilot Mixed Methods Appraisal Tool (MMAT) for systematic mixed studies review. Int J Nurs Stud 2012;49:47-53.

18. Whittemore R, Knafl K. The integrative review: updated methodology. J Adv Nurs 2005;52:546-53.

19. Kavanagh J, Campbell F, Harden A, et al. Mixed methods synthesis: a worked example. In: Hannes K, Lockwood C, eds. Synthesizing qualitative research: choosing the right approach. Chichester, UK: John Wiley \& Sons, 2012:113-36.

20. Fetters MD, Curry LA, Creswell JW. Achieving integration in mixed methods designs-principles and practices. Health Serv Res 2013;48:2134-56. 